\title{
Restoration of sagittal alignment in high-grade isthmic spondylolisthesis using the reverse Bohlman technique with anterior lumbar interbody fusion using a hyperlordotic cage at L4-5: illustrative case
}

\author{
Terrence Ishmael, MBBS, ${ }^{1}$ Vincent Arlet, $\mathrm{MD},{ }^{2}$ and Harvey Smith, $\mathrm{MD}^{2}$ \\ ${ }^{1}$ Department of Orthopedic Surgery, Robert Wood Johnson University Hospital, New Brunswick, New Jersey; and ${ }^{2}$ Department of Orthopedic Surgery, University of \\ Pennsylvania, Philadelphia, Pennsylvania
}

\begin{abstract}
BACKGROUND Circumferential fusion with or without reduction is the preferred treatment for high-grade isthmic spondylolisthesis. Reduction presents significant risk of neurological injury. The authors present one case in which the "reverse Bohlman" technique was used with the addition of a hyperlordotic interbody cage at L4-5 as a means to correct sagittal malalignment while avoiding the reduction of L5 on S1.

OBSERVATIONS The patient was a 22-year-old woman with a long-term history of lower back pain and bilateral L5 radiculopathy secondary to highgrade isthmic lumbar spondylolisthesis. She underwent anterior lumbar interbody fusion using the reverse Bohlman technique plus a hyperlordotic interbody cage at L4-5, followed by decompression and posterior spinal instrumentation and fusion from $L 4$ to the pelvis. At 2-year follow-up, she was found to have complete resolution of symptoms with clinical and radiographic evidence of fusion. Her spinopelvic parameters had significantly improved.

LESSONS The reverse Bohlman technique with the addition of a hyperlordotic interbody cage at L4-5 is a potential alternative treatment method to correct sagittal malalignment while avoiding possible injury to the L5 nerve roots that can be seen in the reduction of high-grade isthmic spondylolisthesis.
\end{abstract}

https://thejns.org/doi/abs/10.3171/CASE208

KEYWORDS high-grade spondylolisthesis; hyperlordotic; anterior lumbar interbody fusion; ALIF

Symptomatic high-grade (Meyerding grade 3 and higher) isthmic spondylolisthesis represents a difficult treatment problem. Patients often have severe lower back pain as well as neurological symptoms. Additionally, they may develop coronal and sagittal deformities. Sagittal malalignment has been associated with increased disability in adults. ${ }^{1,2}$ The decision to treat high-grade spondylolisthesis is multifactorial and could result in severe complications.,

The surgical treatment of high-grade spondylolisthesis is associated with a variable risk of neurological injury; the rate has been reported at $2 \%$ after in situ fusion versus $10 \%$ after reduction and fusion. ${ }^{3}$ Total overall complications in that series were reported as $8 \%$ after in situ fusion and $14 \%$ after reduction. Older series reported up to $20 \%$ rates of pseudoarthrosis, but this was prior to modern fixation techniques. ${ }^{5}$ We present a modification of a previously described technique that improves sagittal alignment while avoiding the risks involved in the reduction of high-grade spondylolisthesis.

\section{Illustrative Case}

The patient was a 22-year-old female who presented with a long history of lower back pain; she had been diagnosed with scoliosis at 10 years of age by an outside physician. Bracing had been recommended, and the patient was then lost to follow-up. Her pain worsened at 16 years of age after a cesarean section. She was treated symptomatically by her primary care physician but later developed radicular symptoms in the L5 distribution bilaterally. She had no change in bowel or bladder function.

The patient's physical examination revealed an obese female with right trunk shift, waist asymmetry, and positive sagittal balance. She had 4/5 strength in the bilateral tibialis anterior and extensor hallucis longus muscles.

ABBREVIATIONS ALIF = anterior lumbar interbody fusion; $\mathrm{BMI}=$ body mass index; $\mathrm{CT}=$ computed tomography; $\mathrm{MRI}=$ magnetic resonance imaging; SVA = sagittal vertical axis; TLIF = transforaminal lumbar interbody fusion.

INCLUDE WHEN CITING Published June 28, 2021; DOI: 10.3171/CASE208.

SUBMITTED September 10, 2020. ACCEPTED March 23, 2021.

(c) 2021 The authors, CC BY-NC-ND 4.0 (http://creativecommons.org/licenses/by-nc-nd/4.0/). 


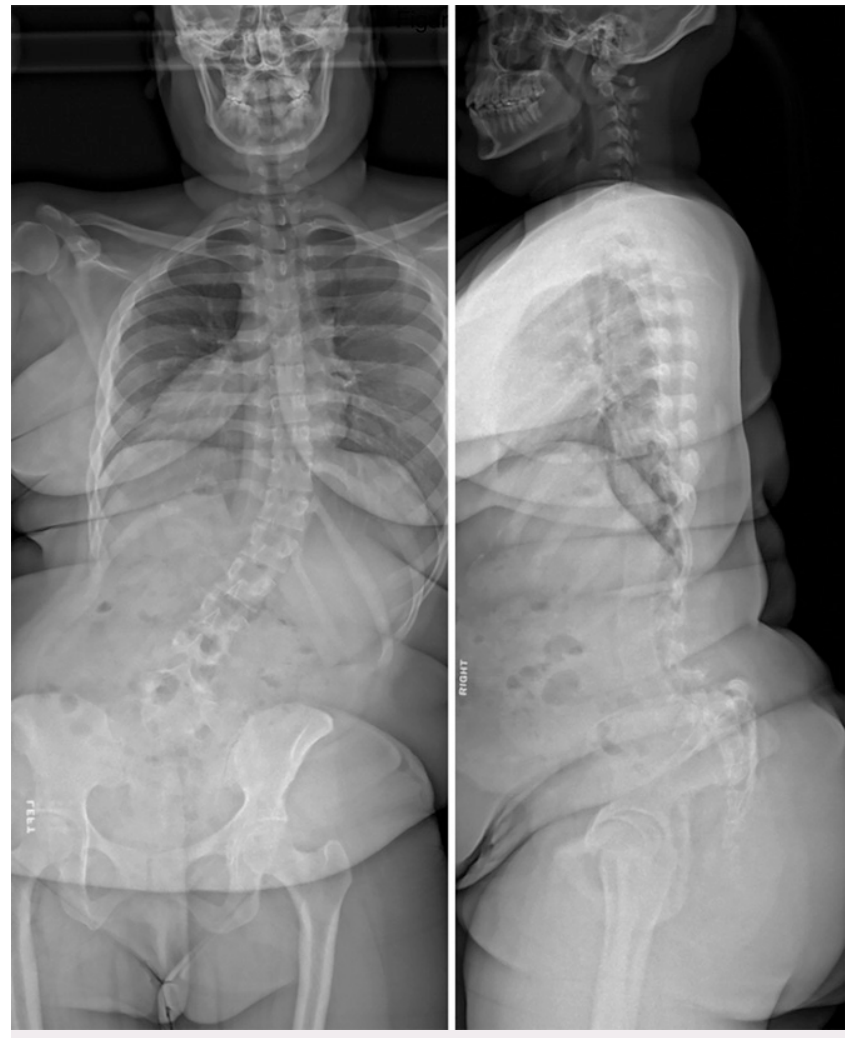

FIG. 1. Standing posteroanterior and lateral radiographs. Left lumbosacral scoliosis $35^{\circ}$, right thoracic scoliosis $37^{\circ}$. Coronal imbalance 79 $\mathrm{mm}$. Spondyloptosis L5-S1. Pelvic incidence $54^{\circ}$, lumbar lordosis $13^{\circ}$ pelvic tilt $35^{\circ}$, sacral slope $20^{\circ}$, thoracic kyphosis $5^{\circ}$, sagittal vertical axis $+90 \mathrm{~mm}$, and L4-S1 lordosis $11^{\circ}$.

Radiographs at initial presentation revealed high-grade isthmic spondylolisthesis (spondyloptosis) of L5 on S1 with a left lumbosacral scoliosis of $35^{\circ}$ and a compensatory right thoracic scoliosis that measured $37^{\circ}$. The patient also had negative coronal balance of $79 \mathrm{~mm}$,
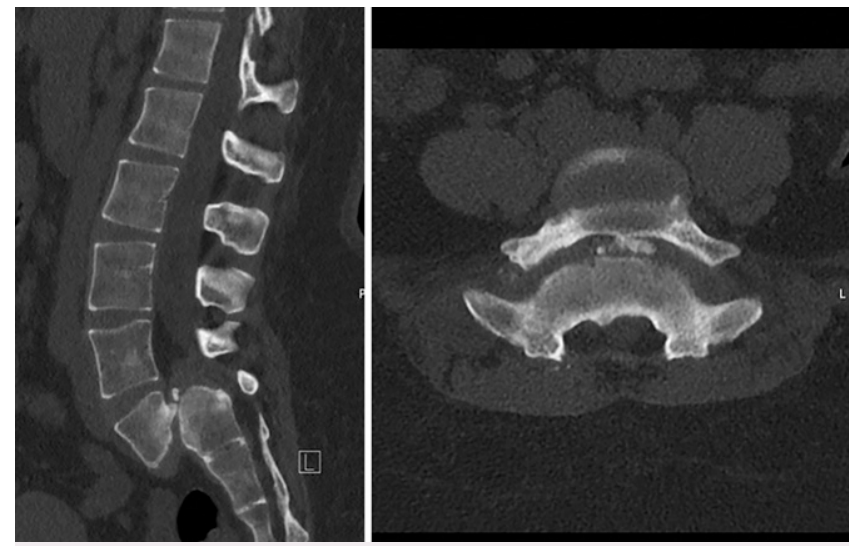

FIG. 2. CT of the mid-saggital and axial lumbar spine showing spondyloptosis of L5-S1.

positive sagittal vertical alignment of $90 \mathrm{~mm}$, pelvic incidence of $54^{\circ}$, lumbar lordosis of $85^{\circ}$, pelvic tilt of $37^{\circ}$, sacral slope of $20^{\circ}$, thoracic kyphosis of $5^{\circ}$, and L4-S1 lordosis of $11^{\circ}$ (Fig. 1). Flexion and extension images of the lumbar spine failed to reveal any motion at the lumbosacral junction. Computed tomography (CT) and magnetic resonance imaging (MRI) of the lumbar spine were performed, which showed mild canal stenosis at L5-S1 and severe foraminal stenosis at the same level (Figs. 2 and 3 ).

In light of the patient's morbid obesity (body mass index [BMI] $45 \mathrm{~kg} / \mathrm{m}^{2}$ ), she was referred to the bariatric surgery service for evaluation. She subsequently underwent a robotic sleeve gastrectomy. She then re-presented to the spine service for surgery 7 months after her bariatric procedure, having lost $35 \mathrm{lb}\left(\mathrm{BMl} 40 \mathrm{~kg} / \mathrm{m}^{2}\right)$ and with worsening lower back and lower extremity complaints.

The patient underwent anterior lumbar interbody fusion (ALIF) at L4-5 with a "reverse Bohlman" interbody fusion between L5 and $\mathrm{S} 1$, followed by a posterior decompression at L4-5 and L5-S1 with instrumentation from $L 4$ to the pelvis. The anterior approach was
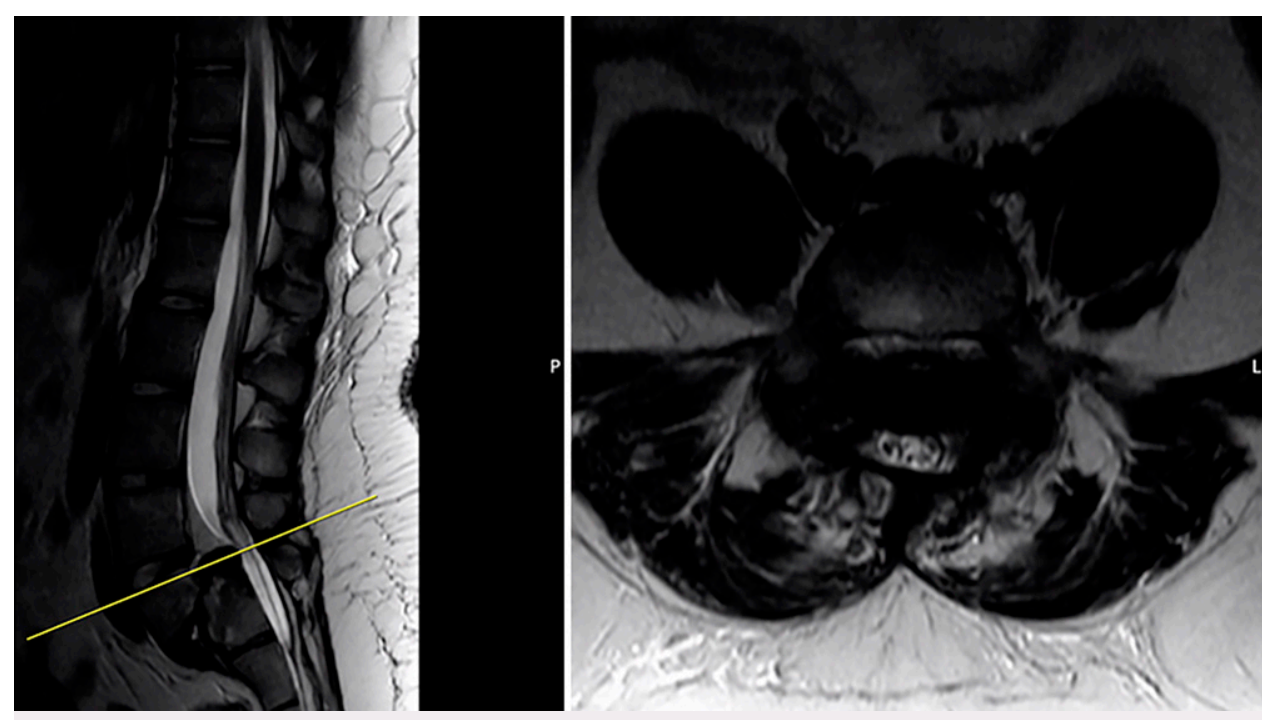

FIG. 3. MRI of the lumbar spine showing marked L5-S1 foraminal stenosis. Mid-sagittal cut and axial cut at level of L5-S1 disc (yellow line). 
performed through a pararectal retroperitoneal route. The L4-5 disc was identified. The aortic bifurcation was then mobilized and retracted. A complete discectomy of $L 4-5$ was then performed. The disc space was then templated to accept a $30^{\circ}$ hyperlordotic cage (NuVasive). Orthogonal fluoroscopic images were obtained, which confirmed good position of the template.

Next, the reverse Bohlman procedure was performed from the superior aspect of the $L 5$ endplate by inserting a guidewire from the superior endplate of L5 into S1 under direct lateral fluoroscopy. A 10-mm-diameter anterior cruciate ligament reamer (Smith and Nephew) was then used to ream over the guidewire from $L 5$ into the body of S1. This was done under fluoroscopic guidance. Great care was taken to avoid destabilizing the guidewire; only the final reamer was driven over its entire length. An appropriately sized fibular allograft was selected and shaped. The leading edge of the graft was contoured using a high-speed burr to facilitate insertion. The graft was then soaked in radiopaque dye to improve fluoroscopic visualization.

The proud portion of the allograft was trimmed to ensure that it remained flush with the superior endplate of $L 5$. The previously selected $30^{\circ}$ hyperlordotic interbody cage was then inserted into the L4-5 disc space.

Once the anterior portion of the case was completed, the patient was repositioned, and the posterior portion of the procedure was commenced. Pedicle screws were placed bilaterally at $L 4$, transsacral

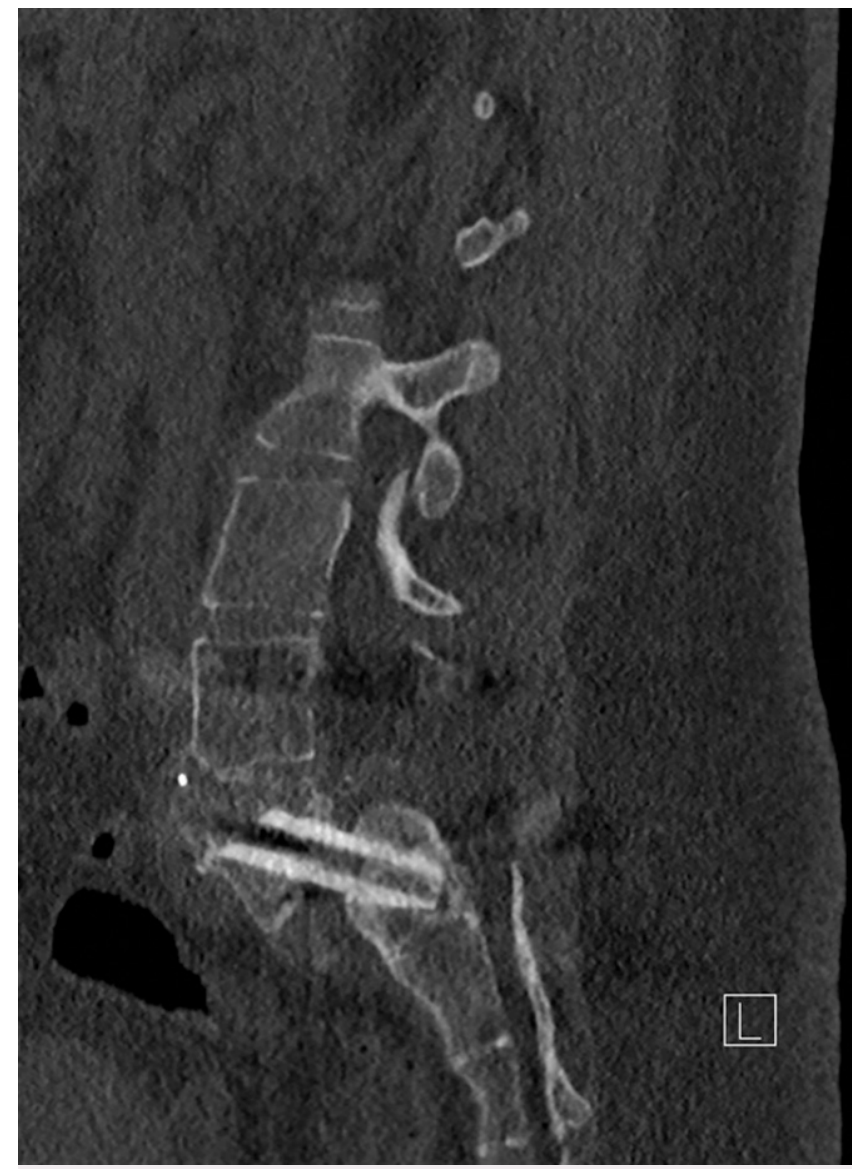

FIG. 4. Midsagittal CT cut demonstrating the fibular strut graft within the bodies of L5 and S1.
S1 screws were placed into the body of $L 5$, and a single right iliac screw for pelvic fixation was used. A wide and complete decompression of the neural elements was performed from L4 to $S 1$. The course of bilateral L5 nerve roots was followed to ensure that no compression was present. There were no changes recorded on intraoperative neuromonitoring, and the patient's bilateral L5 radiculopathy was greatly improved upon reversal of anesthesia.

The entire procedure, including anesthetic time and positioning, lasted 424 minutes. The surgical time for the anterior procedure was approximately 180 minutes. Repositioning took 30 minutes, and the posterior portion of the case lasted $150 \mathrm{mi}-$ nutes. Estimated blood loss was $400 \mathrm{ml}$. No postoperative immobilization was used.

The patient's immediate postoperative course was uneventful. Standing lumbar radiographs and a CT scan of the lumbar spine were obtained prior to discharge (Fig. 4). The patient was discharged to home on postoperative day 3. She was then seen 2 weeks after surgery for routine staple removal. Fifteen months after surgery, the patient gave birth to her second child via a routine cesarean section. At the time of her 2-year postoperative visit, the patient had returned to work full time. All of her back pain and radicular symptoms had resolved. Her lumbar lordosis had improved from $13^{\circ}$ to $57^{\circ}$, and her sagittal vertical axis (SVA) had improved from $+90 \mathrm{~mm}$ to $+46 \mathrm{~mm}$, with near-complete correction of her scoliosis (Fig. 5).

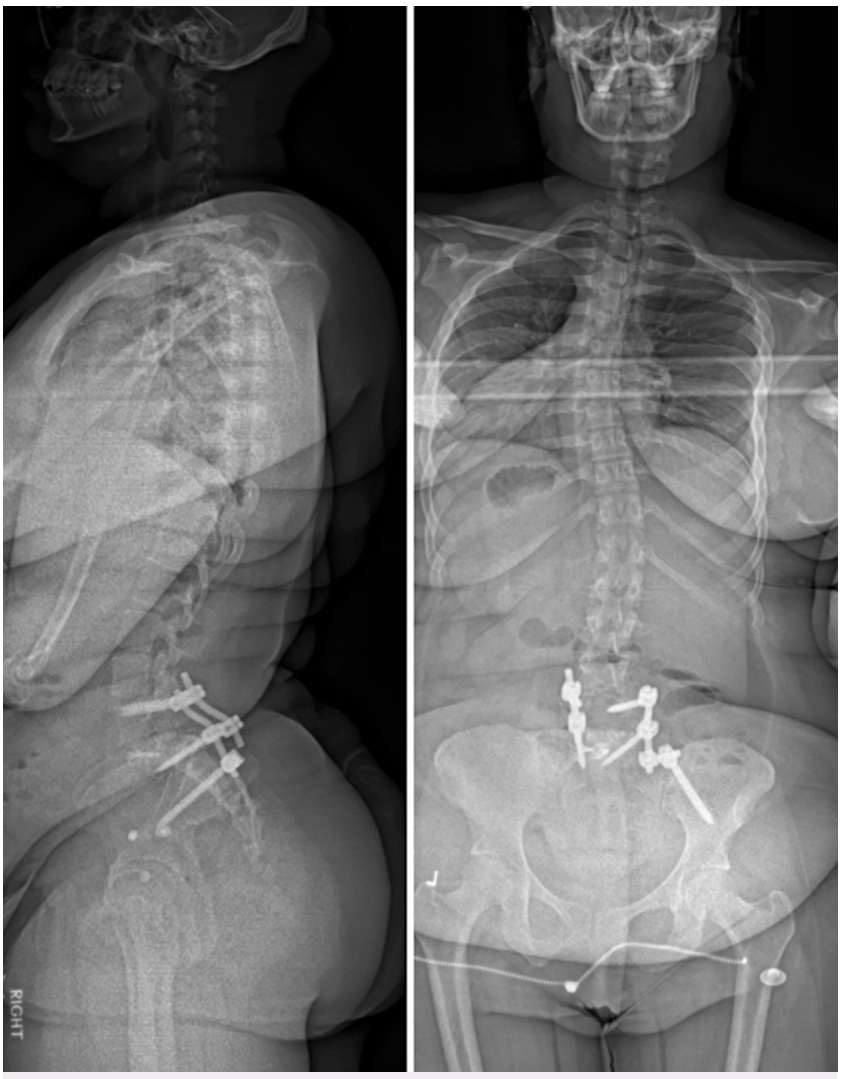

FIG. 5. Standing posteroanterior and lateral radiographs two years after surgery, the patient had no complaints. Her back pain was completely resolved. Sagittal and coronal alignment improved significantly. Pelvic incidence $46^{\circ}$, lumbar lordosis $57^{\circ}$, pelvic tilt $14^{\circ}$, sacral slope $33^{\circ}$, sagittal vertical axis $+46 \mathrm{~mm}$, and L4-S1 lordosis $35^{\circ}$. 
Two key technical points for this procedure are (1) contouring of the fibula strut graft to ensure that it could easily be inserted into the bodies of $L 5$ and $S 1$ and remained recessed below the superior endplate of $\mathrm{L} 5$ and (2) avoiding reaming over the terminal $5 \mathrm{~mm}$ of the guidewire, which would destabilize the pin and create an eccentric tract.

\section{Discussion}

\section{Observations}

Surgical treatment of high-grade isthmic spondylolisthesis and spondyloptosis is very challenging. Several techniques have been proposed in the literature, each with its own pros and cons. In situ fusion via posterolateral fusion and cast immobilization has been reported to have a high rate of pseudoarthrosis up to $44 \%{ }^{6}$ Schoenecker et al. also reported the development of cauda equina syndrome in 12 patients (6\%) after in situ arthrodesis for high-grade spondylolisthesis. ${ }^{7}$ In addition, in situ fixation did not address the often present sagittal malalignment.

Boxall et al. reported that $18 \%$ of patients in their series who had undergone decompression and in situ fusion developed pseudoarthrosis. ${ }^{6}$ There were no reported permanent neurological deficits.

Bradford and Boachie-Adjei reported their experience with anterior/ posterior reduction and stabilization. ${ }^{5}$ Extensive posterior decompression (including sacral dome osteotomy) and posterolateral fusion were performed. Their patients were placed in halo-femoral or halo-pelvic traction and the spondylolisthesis were gradually reduced with traction. After 7-10 days, a second-stage procedure was performed using an anteriortransperitoneal approach with interbody fusion with a fibula strut graft. The patients were then placed in a body cast incorporating traction pins. The authors reported an $18 \%$ rate of pseudoarthrosis, a $6 \%$ rate of cauda equina syndrome, a $6 \%$ rate of pin site infection, and an $11 \%$ rate of superior mesenteric artery syndrome. At the final follow-up, no permanent neurological deficits were reported.

Gaines et al. reported on their technique of resection of $L 5$ and reducing $L 4$ to $S 1^{8,9}$ In their initial series, they reported that neither of the patients in their series had permanent neurological deficits. In their second series, $25 \%$ of the patients had permanent neurological deficits, with $75 \%$ having early neurological deficits. Of those with permanent deficits, the vast majority had preoperative neurological deficits.

Reduction with transforaminal lumbar interbody fusion (TLIF) between L5 and S1 plus instrumented posterior spinal fusion from L4 to $\mathrm{S} 1$ has become a commonly used tool in the management of high-grade isthmic spondylolisthesis. This has also been associated with a high rate of neurological injury, specifically of the L5 nerve root because it is stretched during the reduction maneuver. Schär et al. reported a $29 \%$ rate of $L 5$ radiculopathy, with 4 of the 5 patients having complete resolution within 3 months of surgery, while the fifth was lost to follow-up. ${ }^{10}$ In their series, Ferrero et al. reported a $70 \%$ rate of postoperative neurological complications, the majority of which resolved within 3-6 months of surgery. ${ }^{11}$

The Bohlman technique and its various modifications, including the reverse Bohlman technique, have gained traction over the last several years, ${ }^{12-16}$ with varying results. For the most part, these techniques have involved decompression and in situ fusion, with little improvement in overall sagittal alignment. As discussed previously, sagittal malalignment is associated with increased disability in adults. ${ }^{1}$ Bartolozzi et al. reported on 15 patients with decompression, partial reduction, and fusion, with $33 \%$ neurological deficits (3 partial unilateral L5 motor deficits and 2 L5 sensory deficits). ${ }^{17}$ Overall sagittal alignment was not evaluated.

\section{Lessons}

The technique described in this case report addresses overall sagittal alignment while avoiding the possible complications associated with attempted reduction of the spondyloptosis or resection as proposed by Gaines. This is achieved by the addition of an ALIF at the L4-5 level with a hyperlordotic cage to the reverse Bohlman technique for the management of high-grade spondylolisthesis.

This procedure could potentially be addressed from an all-posterior approach using the Bohlman technique with multiple TLIFs and posterior column osteotomies or a three-column osteotomy, likely pedicle subtraction osteotomy, in the lumbar spine. Either of these options could avoid the potential morbidity of an anterior surgical approach while providing sagittal plane correction. Multiple TLIFs would have to be performed in order to obtain the same correction obtained with a single ALIF at L4-5. This would require greater proximal instrumentation and fusion, but it is a reasonable option. The addition of a three-column osteotomy would add another level of complexity and morbidity to this procedure that we believed was not desirable.

Percutaneous posterior instrumentation placement could be combined with the anterior approach used in this patient. This is commonly used in combined anterior/posterior spinal deformity correction. Purely percutaneous posterior techniques would not address the unstable Gill fragment associated with high-grade isthmic spondylolisthesis. Percutaneous instrumentation combined with a "mini-open decompression" is a modification that could be effectively used.

The senior authors have extensive experience with using hyperlordotic interbody cages for correction of sagittal plane deformity. In their retrospective review in 2016, they recorded a mean of $29^{\circ}$ of sagittal plane correction using $30^{\circ}$ hyperlordotic cages. This decreased to $19^{\circ}$ in the presence of spondylolisthesis. The mean lumbar lordosis increased from $39^{\circ}$ to $59^{\circ}$, and the mean SVA reduced from $113 \mathrm{~mm}$ (range $38-320 \mathrm{~mm}$ ) to $43 \mathrm{~mm}$ (range -13 to 112 $\mathrm{mm}){ }^{18}$ This demonstrates the power of hyperlordotic interbody cages when used in sagittal plane deformity correction.

This technique is not without risk. Anterior approaches to the lumbar spine have been associated with visceral and vascular injuries, reported at a rate as high as $38.3 \%$. $^{19}$ These can range from wound complications to vascular injuries. Retrograde ejaculation has been reported to range from $0.4 \%^{20}$ to $8 \%{ }^{21}$ in two commonly cited series. In our experience, however, these complications are rare, but this may give one pause when considering the treatment of younger male patients.

In addition, this is a single case report. Further study of patients treated with this technique would be required to evaluate it against other techniques.

In conclusion, the reverse Bohlman technique with the addition of a hyperlordotic interbody cage at $L 4-5$ is a viable alternative to treat high-grade lumbar spondylolisthesis through fusion of L5-S1 and correction of sagittal malalignment while avoiding neurological complications associated with the reduction of L5 on S1 or resection of $\mathbf{L} 5$.

\section{References}

1. Glassman SD, Bridwell K, Dimar JR, et al. The impact of positive sagittal balance in adult spinal deformity. Spine (Phila Pa 1976). 2005;30(18):2024-2029.

2. Harroud A, Labelle H, Joncas J, Mac-Thiong JM. Global sagittal alignment and health-related quality of life in lumbosacral spondylolisthesis. Eur Spine J. 2013;22(4):849-856. 
3. Fu KM, Smith JS, Polly DW Jr, et al. Morbidity and mortality in the surgical treatment of six hundred five pediatric patients with isthmic or dysplastic spondylolisthesis. Spine (Phila Pa 1976). 2011; 36(4):308-312.

4. Mac-Thiong JM, Hresko MT, Alzakri A, et al. Criteria for surgical reduction in high-grade lumbosacral spondylolisthesis based on quality of life measures. Eur Spine J. 2019;28(9):2060-2069.

5. Bradford DS, Boachie-Adjei O. Treatment of severe spondylolisthesis by anterior and posterior reduction and stabilization. A long-term follow-up study. J Bone Joint Surg Am. 1990;72(7):1060-1066.

6. Boxall D, Bradford DS, Winter RB, Moe JH. Management of severe spondylolisthesis in children and adolescents. J Bone Joint Surg Am. 1979;61(4):479-495.

7. Schoenecker PL, Cole HO, Herring JA, et al. Cauda equina syndrome after in situ arthrodesis for severe spondylolisthesis at the lumbosacral junction. J Bone Joint Surg Am. 1990;72(3):369-377.

8. Gaines RW, Nichols WK. Treatment of spondyloptosis by two stage L5 vertebrectomy and reduction of L4 onto S1. Spine (Phila Pa 1976). 1985;10(7):680-686.

9. Lehmer SM, Steffee AD, Gaines RW Jr. Treatment of L5-S1 spondyloptosis by staged $L 5$ resection with reduction and fusion of $L 4$ onto S1 (Gaines procedure). Spine (Phila Pa 1976). 1994;19(17): 1916-1925.

10. Schär RT, Sutter M, Mannion AF, et al. Outcome of L5 radiculopathy after reduction and instrumented transforaminal lumbar interbody fusion of high-grade L5-S1 isthmic spondylolisthesis and the role of intraoperative neurophysiological monitoring. Eur Spine $J$. 2017;26(3):679-690.

11. Ferrero $E$, Ilharreborde $B$, Mas V, et al. Radiological and functional outcomes of high-grade spondylolisthesis treated by intrasacral fixation, dome resection and circumferential fusion: a retrospective series of 20 consecutive cases with a minimum of 2 years follow-up. Eur Spine J. 2018;27(8):1940-1948.

12. Smith MD, Bohlman HH. Spondylolisthesis treated by a single-stage operation combining decompression with in situ posterolateral and anterior fusion. An analysis of eleven patients who had long-term follow-up. J Bone Joint Surg Am. 1990;72(3):415-421.

13. Roca J, Ubierna MT, Cáceres E, Iborra M. One-stage decompression and posterolateral and interbody fusion for severe spondylolisthesis. An analysis of 14 patients. Spine (Phila Pa 1976). 1999;24(7):709-714.
14. Smith JA, Deviren V, Berven S, et al. Clinical outcome of trans-sacral interbody fusion after partial reduction for high-grade L5-S1 spondylolisthesis. Spine (Phila Pa 1976). 2001;26(20):2227-2234.

15. Hanson DS, Bridwell KH, Rhee JM, Lenke LG. Dowel fibular strut grafts for high-grade dysplastic isthmic spondylolisthesis. Spine (Phila Pa 1976). 2002;27(18):1982-1988

16. Macagno AE, Hasan S, Jalai CM, et al. "Reverse Bohlman" technique for the treatment of high grade spondylolisthesis in an adult population. J Orthop. 2016;13(1):1-9.

17. Bartolozzi P, Sandri A, Cassini M, Ricci M. One-stage posterior decompression-stabilization and trans-sacral interbody fusion after partial reduction for severe L5-S1 spondylolisthesis. Spine (Phila Pa 1976). 2003;28(11):1135-1141.

18. Saville PA, Kadam AB, Smith HE, Arlet V. Anterior hyperlordotic cages: early experience and radiographic results. J Neurosurg Spine. 2016;25(6):713-719.

19. Rajaraman V, Vingan $R$, Roth $P$, et al. Visceral and vascular complications resulting from anterior lumbar interbody fusion. J Neurosurg. 1999;91(suppl 1):60-64.

20. Flynn JC, Price CT. Sexual complications of anterior fusion of the lumbar spine. Spine (Phila Pa 1976). 1984;9(5):489-492.

21. Christensen FB, Bünger CE. Retrograde ejaculation after retroperitoneal lower lumbar interbody fusion. Int Orthop. 1997;21(3): 176-180.

\section{Disclosures}

Dr. Arlet reports receiving personal fees from DePuy Synthes, Zimmer Biomet, and NuVasive.

\section{Author Contributions}

Conception and design: all authors. Acquisition of data: Ishmael, Smith. Analysis and interpretation of data: Ishmael, Smith. Drafting the article: Ishmael. Critically revising the article: Ishmael, Smith. Reviewed submitted version of manuscript: Ishmael, Smith. Approved the final version of the manuscript on behalf of all authors: Ishmael. Administrative/technical/material support: Ishmael. Study supervision: Arlet.

\section{Correspondence}

Terrence Ishmael: Pediatric Orthopedic Associates, East Brunswick, NJ. terry.ishmael@gmail.com. 